\title{
Cerebral organoids: ethical issues and consciousness assessment
}

\author{
Andrea Lavazza, ${ }^{1}$ Marcello Massimini ${ }^{2,3}$
}

\begin{abstract}
${ }^{1}$ Centro Universitario Internazionale, Arezzo, Italy ${ }^{2}$ Department of Biomedical and Clinical Sciences "Luigi Sacco", University of Milan, Milan, Italy' ${ }^{3}$ IRCCS Fondazione Don Gnocchi Onlus, Milan, Italy
\end{abstract}

\section{Correspondence to}

Dr Andrea Lavazza, Centro Universitario Internazionale, via Garbasso 42, Arezzo 52100, Italy; lavazza67@gmail.com

Received 2 September 2017 Revised 8 January 2018 Accepted 5 February 2018 Published Online First 28 February 2018

\section{Linked}

- http://dx.doi.org/10.1136/ medethics-2018-104778

- http://dx.doi.org/10.1136/ medethics-2018-104976

Check for updates

To cite: Lavazza A, Massimini M. J Med Ethics 2018:44:606-610.

\section{ABSTRACT}

Organoids are three-dimensional biological structures grown in vitro from different kinds of stem cells that selforganise mimicking real organs with organ-specific cell types. Recently, researchers have managed to produce human organoids which have structural and functional properties very similar to those of different organs, such as the retina, the intestines, the kidneys, the pancreas, the liver and the inner ear. Organoids are considered a great resource for biomedical research, as they allow for a detailed study of the development and pathologies of human cells; they also make it possible to test new molecules on human tissue. Furthermore, organoids have helped research take a step forward in the field of personalised medicine and transplants. However, some ethical issues have arisen concerning the origin of the cells that are used to produce organoids (ie, human embryos) and their properties. In particular, there are new, relevant and so-far overlooked ethical questions concerning cerebral organoids. Scientists have created so-called mini-brains as developed as a few-monthsold fetus, albeit smaller and with many structural and functional differences. However, cerebral organoids exhibit neural connections and electrical activity, raising the question whether they are or (which is more likely) will one day be somewhat sentient. In principle, this can be measured with some techniques that are already available (the Perturbational Complexity Index, a metric that is directly inspired by the main postulate of the Integrated Information Theory of consciousness), which are used for brain-injured non-communicating patients. If brain organoids were to show a glimpse of sensibility, an ethical discussion on their use in clinical research and practice would be necessary.

\section{INTRODUCTION: FROM THOUGHT TO LAB EXPERIMENTS}

In a famous mental experiment, philosopher Hilary Putnam imagined a cruel scientist able to dislodge the brain from one's body and immerse it in a vat of nutrient fluid to keep it alive. ${ }^{1}$ The nerve terminals are then connected to a supercomputer, so that the brain's owner is under the illusion that everything is perfectly normal. The person will think that there are objects and people in an environment and will have feelings and perceptions that correspond to the actions that he or she will conceive. But all that the person (the brain) feels is the result of the pulses transmitted by the computer to the nerve terminals.

This scenario was of course constructed by a philosopher, under no claim of being realistic, in order to theoretically discuss the themes of scepticism and knowledge. For some years, however, the idea of a brain in a vat has no longer been just a mental experiment, but also a laboratory experiment. For a decade now, scientists have been trying to build mammal and human organs starting from single cells: the so-called organoids. ${ }^{2}$ Recently, scientists have also tried to produce cerebral organoids. ${ }^{3}$

The latter are by no means similar to adult brains, nor is technology able, for now, to simulate an external reality and a body, transmitting it to the brain with the relative perceptions and feelings. However, the rapid progress that is being made suggests that one should begin to reason on the ethical aspects of organoids in general, and cerebral organoids in particular. ${ }^{4-6}$ After a discussion of what cerebral organoids are, in this paper we will address some new, relevant ethical issues related to the (today still remote) possibility of creating a sentient system in a dish. Then we will discuss the need to find shared criteria (which is not easy) and to establish a threshold beyond which simple biological material becomes something that should not be manipulated or destroyed, at least not without having a serious ethical discussion on the matter. Specifically, we want to focus on the developmental states in which a cerebral organoid may begin to be capable of experience, potentially including pain sensations.

To do this, we need a general theory of consciousness that attempts to explain what experience is and what type of physical systems can have it. ${ }^{7}$ The Integrated Information Theory (IIT) does so starting from phenomenological axioms to derive postulates about the properties required of physical mechanisms to support consciousness. The fundamental phenomenological axioms are (i) that conscious experience is informative (each conscious experience differs in its own specific way from countless other possible experiences) and (ii) that conscious experience is integrated (each conscious experience cannot be split). The corresponding fundamental postulate is that a system has subjective experience to the extent that it is capable of integrating information.

The ability to experience depends on an optimal balance between diversity (information) and unity (integration), a non-trivial condition for a physical systems. ${ }^{8} 9$ Importantly, the theory proposes a theoretical measure (PHI), as well as related empirical metrics, to quantify a system's capacity for integrating information. Although there is general consensus on the idea that consciousness is tied to the capacity of different cortical areas to 
share information, IIT seems to be specifically relevant in the case of cerebral organoids for at least two reasons. ${ }^{10} 11$ First, because it explicitly predicts that consciousness can be graded among biological organisms. ${ }^{12}$ Second, because it postulates that consciousness can be generated independently of sensory processing, executive functions or motor behaviour, if the internal architecture of the system is intrinsically optimised for integrating information. ${ }^{13}$

\section{ORGANOIDS, CEREBRAL ORGANOIDS AND ETHICAL ISSUES}

Thanks to pluripotent stem cells (embryonic stem cells and induced pluripotent stem cells) and organ-restricted adult stem cells, research on so-called organoids is guided by the desire to obtain three-dimensional (3D) models of tissues and organs for basic research. Another goal is to get closer to the so-called Holy Grail of Medical Science, that is, making it possible to transplant organs while overcoming problems of scarcity, compatibility and rejection. $^{14}$

Organoids are biological entities produced in vitro from stem cells whose differentiation can be oriented towards the typical organisation (architecture and physiology) of a human adult organ within a specially prepared environment: usually Matrigel (a mixture of protein from mouse sarcomas) and an adequate scaffolding (made by 3D support matrices). The denomination of 'organoid' comes from the fact that one can currently only obtain miniaturised and simplified versions of an organ, although often endowed with many of its desired structural and functional features.

It could be said that an organoid in a $3 \mathrm{D}$ in vitro culture has to 'replicate not only the complexity of the cell types present in the organ, and the processes of self-organization of the tissue, but also the main organization of the whole organ; in the case of brain organoids, "the appearance of different brain regions"'. ${ }^{15}$ The key aspects are self-assembly and differentiation. ${ }^{16} 17$ Today we have organoids of the retina, intestine, kidney, pancreas, liver, inner ear, thyroid and so forth. However, the main ethical issues seem to be related to brain organoids. 'Human brain organoids are stem cell-derived 3D tissues that self-assemble into organized structures that resemble the developing human brain'. ${ }^{18}$ They include cerebral organoids and region-specific organoids. The seminal study in this field is due to Lancaster et al, who started with human adult skin cells reprogrammed as induced pluripotent stem cells so as to create a brain organoid as a model for the study of microcephaly. ${ }^{3}$

Two-dimensional cultures had already produced neurons capable of producing action potentials and synapses, as well as of integrating among cortical neurons of mice. Recently, great steps forward have been made in 3D self-organising cultures, thanks to extracellular matrices. The study by Lancaster $e t$ al has shown distinct and interdependent brain regions with connections via interneurons and a high level of similarity on a cellular level. ${ }^{3}$ Organoids of about $4 \mathrm{~mm}$ replicated in vitro the in vivo development at least up to the late mid-fetal period (19-24 weeks of gestation), with differences in gene expression. ${ }^{19}$ Qian et al have obtained neurons corresponding to all six layers of the cerebral cortex, ${ }^{20}$ but they still have not fully developed stable synapses or circuitry. And in general, organoids have a nucleus of cells that tend to rot in a short time due to lack of vascularity, and are devoid of surrounding embryonic tissues, glial cells, meninges and immune cells. Organoid models are also limited by the great variability among organoids and the absence of predefined axis.

Lancaster and Knoblich have described a protocol for 'generating $3 \mathrm{D}$ brain tissue (...) which closely mimics the endogenous developmental program. This method can easily be implemented in a standard tissue culture room, and can give rise to developing cerebral cortex, ventral telencephalon, choroid plexus and retinal identities, among others, within 1-2 months'.$^{21}$ But there are also layers of the cortex, the hippocampus (a crucial area for memory) and the spinal cord. And organoids, according to the authors, can be maintained for more than a year in long-term cultures.

Kelava and Lancaster claim that human pluripotent stem cells can be used to produce 'organoids which faithfully recapitulate, on a cell-biological and gene expression level, the early period of human embryonic and fetal brain development'. ${ }^{15}$ In this vein, Birey et al produced 'three-dimensional spheroids from human pluripotent stem cells that resemble either the dorsal or ventral forebrain and contain cortical glutamatergic or GABAergic neurons', thus recapitulating the saltatory migration of interneurons in the fetal forebrain. ${ }^{22}$ They also showed that after migration, interneurons functionally integrate with glutamatergic neurons to form a microphysiological system. And 'spheroids cells were remarkably similar to those from corresponding regions of humans fetal brain', with 'both excitatory and inhibitory neuronal activity'. ${ }^{23}$

Brain organoids are still unable to reproduce an in vivo brain, but attempts are being made to solve so-called plumbing and scaffolding problems, that is, how to bring oxygen and nutrients (so as to keep the cells alive) and grow organoids beyond the current millimetre scale. However, the so-called mini-brains that grow in vitro, isolated from a complete embryo and without interaction with the environment, may not be able to fully develop as happens in vivo.

The production of a cerebral organoid with a degree of development comparable to a few-months-old embryo is probably one of the greatest breakthroughs in biology: together with cell reprogramming technology and gene editing methods it allows to reach frontiers that were unthought-of even just a few years ago. Therefore, it is hard to underestimate the importance of organoids in general and brain organoids in particular. The latter have already allowed for a study of microcephaly related to the Zika virus, but they will also be used for autism, Parkinson's disease, multiple sclerosis, Alzheimer's disease, epilepsy, traumatic brain injuries and also in neuro-oncology. ${ }^{24}$

So, it is likely that investments and researches on the topic will increase, leading to knowledge and techniques able to create more 'evolved' mini-brains. In particular, the direction will probably be that of bigger organoids to hopefully achieve organ-level functionality. In any case, the use of brain organoids is starting to find commercial applications. Indeed, Hubrecht Organoid Technology (HUB) and the health insurance companies CZ, Zilveren Kruis and Menzis have recently started a $€ 3$ million validation trial for use of HUB Organoid Technology to test if it can be used to determine the response of patients with cystic fibrosis to new drug therapies. ${ }^{25-27}$

Therefore, from the ethical point of view, the probable scenario is the following. Prima facie, brain organoids can be considered a more acceptable form of experimentation than that on human fetuses (weeks 9 and beyond after fertilisation), animals and voluntary human adults. In fact, no living being is destroyed, damaged or put at risk in tests involving artificial mini-brains, and their level of scientific reliability might soon be equivalent to that of traditional methods. So, there is no efficiency loss to the detriment of the ill awaiting a cure. Also, cerebral organoids can be cheaper- both in terms of material and in terms of working hours-than other forms of experimentation, and therefore their use may make resources available for 
other relevant uses in the medical sector. All of this may lead one to think that the creation and use of brain organoids are non-problematic.

However, there are a number of critical issues that need to be addressed. The 'classical ethical issues' include the use of embryonic stem cells; 'the type of donor consent and ethics review needed for long-term storage and use and for feedback of clinically relevant findings to the patient ${ }^{16}$; the possibility that brain organoids are transferred to chimeric animal models, or connected with other organoids in complexes whose status is unclear and finally the question of patentability of organoids. Here we will focus on a novel ethical quandary, namely consciousness assessment and its potential consequences.

\section{SENTIENT CEREBRAL ORGANOIDS? A WAY TO ASSESS NEW ETHICAL QUANDARIES}

As mentioned, research on cerebral organoids seems to be making rapid progress both for medical applications and for probe human brain evolution and development. ${ }^{28}$ Bigger and more developed mini-brains, with a greater degree of differentiation and internal organisation, could therefore raise the ethical problems associated with the creation of life and/or acquisition of human qualities in research involving human organoids. ${ }^{29}$

It is said that without input and output, the mini-brains' neural networks cannot reach maturity, but the issue is open because 'transcriptional analysis and comparison to the developing human brain revealed that hCSs after 2.5 months resembled the mid-fetal prenatal brain (19-24 post-conception weeks). Cortical neurons were accompanied by a network of nonreactive astrocytes and were synaptically connected'. ${ }^{19}$

Today, lab-made cerebral organoids already 'acquire structural traits of mature neurons, including dendritic spine-like structures' and organoids as old as 8 months showed spikes and excitatory monosynaptic connection with high-density silicon microelectrodes. ${ }^{30}$ 'These results suggest that brain organoids establish neuronal networks that can support self-organized patterns of activity'.30 Also, mini-brains show the differentiation of photoreceptor-like cells endowed with proteins for light responsiveness. These photosensitive cells 'can respond to non-invasive, light based sensory stimulation'. ${ }^{30}$ These advancements indicate that it is possible to transmit to cerebral organoids afferent stimulations that might trigger simple sensations.

Although these studies seem to show rapid and important progress, it may be useful to point out that it will probably take time before it is possible to produce a fully developed human mini-brain in a dish. The claimed similarity between the forebrain neurons of an embryo at a particular developmental stage and the cells of organoids is based on the similarity between transcription factors and other marker genes characteristic for specific cell types. So far, not even the most elementary synaptic circuits-for instance, thalamic relay cells projecting onto cortical glutamatergic neurons to excite them-have been shown to form. And none of the known organoids displays any of the complex electrical signature characteristic of a functioning - and conscious-cortex ${ }^{\mathrm{i}}$.

However, one might wonder whether mini-brains could be somewhat sentient, that is, capable of experiencing pain. In the future, organoids could in fact be developed to the point where they acquire sensory and even potential cognitive functions. This would not be the researchers' primary goal, but it may come as a byproduct of achieving the set research

${ }^{\mathrm{i}}$ We thank a reviewer for this very helpful remark. goals: to study development mechanisms, to test specific treatments, to produce tissues to be transplanted, etc. But since the organoids are devoid of the ability to communicate, we would have no clue about their developmental stage or their capacity to generate sentient internal states. For this reason, there should be tests to assess objectively the capacity for consciousness, irrespectively of sensory inputs, motor outputs and communication.

Although no such test has been devised for cerebral organoids, recent efforts to assess consciousness in brain-injured non-communicating patients may provide, at least, a very rudimentary starting point to tackle the problem. ${ }^{7}$ The challenge of detecting consciousness in brain-injured, comatose patients shares some basic similarities with the issue posed by cerebral organoids. Indeed, much attention has been recently devoted to the development of objective brain-based indices of consciousness that are independent of sensory processing, executive functions and motor outputs. ${ }^{3132}$

One promising approach lies in the Perturbational Complexity Index (PCI), a metric that is directly inspired by the main postulate of IIT, that is, that consciousness relies on the joint presence of integration and differentiation in neural circuits, as explained above in Introduction. Calculating PCI involves locally perturbing the cerebral cortex (with transcranial magnetic stimulation) and measuring the complexity of the electrical response of the rest of the brain (with electroencephalography). ${ }^{33}$ The underlying idea is that PCI should be low if interactions among neural elements are reduced (loss of integration), because the response engaged by transcranial magnetic stimulation (TMS) is spatially restricted; PCI is also low if many interacting areas react to the perturbation in a stereotypical way (loss of differentiation), because in this case the resulting response is large but simple. In fact, PCI should reach high values only if the initial perturbation is transmitted to a large set of neural elements that react in a differentiated way.

As such, being based on general theoretical principles, $\mathrm{PCI}$ is totally independent of sensory processing, executive functions or motor behaviour and can be graded. Since brain-injured, unresponsive patients are fully inaccessible and do not provide any reliable evidence about their state of consciousness, PCI had to be first validated and calibrated on a large benchmark population of subjects who could confirm the presence or absence of conscious experience through reports. Despite some individual variability within this large sample, PCI was lower in all unresponsive subjects who did not report any conscious experience on awakening from non-rapid eye movement (NREM) sleep or midazolam, xenon and propofol anaesthesia, and was invariably higher in conditions in which consciousness was present. ${ }^{34}$

An interesting perspective is that an advanced version of PCI, possibly using finer stimulation and recording techniques (eg, a combination of optogenetic stimulation and calcium imaging) may be developed in the future for cerebral organoids-a version adapted for in vitro cortical slices has been recently tested. ${ }^{35}$ Clearly, the problem would still be how to validate this new index and identify a valid operational cut-off above which we could establish that the cerebral organoid has some capacity for consciousness. As for PCI, the cut-off determination process would, however, have to start from some known points of reference, for example, the values exhibited in the brain of an adult human being across different states (wakefulness, sleep, dreaming, anaesthesia, brain injury) and then gradually move to more challenging cases, such as newborns, primates, rodents and finally, organoids. To the extent that the proposed measurement (say, a novel index of network complexity) is good enough to be 
generalised across species and types of brain circuits, it could at least allow for a coarse comparison on a common scale.

Nevertheless, even if there were an objective measure of consciousness, the ethical scenario would still be unprecedented and complex. Therefore, one can at least sketch some coordinates along accepted facts. In the context of consequentialist ethics, an entity that is sentient (which can feel pain/has a minimum degree of consciousness) may have interests. ${ }^{36}$ And if it has interests, it can be included in the consequentialist calculation of the consequences. Since the organoid would be at the first stage of sentience, it would be appropriate to adopt hedonistic consequentialism, which prescribes to minimise pain as widely understood. However, in a consequentialist perspective the interests should be weighed against each other. On the one hand, we may have the interests of a patient with Alzheimer's disease who is likely to fall into total oblivion, and on the other hand the suffering of an entity comparable to a very simple lifeform that we would usually be willing to sacrifice in the face of the interests of a human being. From the consequentialist perspective, judgement of the rightness or wrongness of the use of sentient brain organoids for research, and especially of brain tissue transplants (when they become possible), should therefore be assessed on the grounds of the expected values of its outcomes, that is, the risk-benefit trade-off or ratio.

At this point, one might wonder what sort of suffering might the organoids experience and whether it is somewhat comparable to the suffering experienced by animal models. These assumptions cannot be verified for now, either behaviourally or instrumentally. In fact, we infer the suffering of animals from some physiological parameters and from their avoidance and signalling behaviours (sound emission, attempts to cure wounds, etc). We now believe that a mammal or an octopus may experience pain and suffering at least as physical discomfort, but we tend to think that insects do not feel pain because wounds or mutilations do not change their behaviour. ${ }^{37}$

Now, when it comes to organoids we do not have any of these parameters. However, if some future objective index could suggest that some human cerebral organoids have some (although very low) capacity for consciousness, such a primitive level of consciousness could allow them to experience forms of suffering somewhat similar to those experienced by individuals left in a behaviourally unresponsive state. ${ }^{38}$ The organoids would not suffer from confinement or deprivation of opportunities for their typical behaviour, as is the case with animals used in labs, but might experience physical pain and discomfort akin to that reported by patients who have been in altered states of consciousness. Obviously, given the purpose of the studies for which organoids were created, they could not be anaesthetised, since analgesia by definition alters the normal brain activity.

These insights come from a consequentialist perspective, but often our moral intuitions are not in tune with consequentialism. From a deontological point of view, although experiments with sentient brain organoids bring many positive effects in terms of care, using brain organoids as a pure means could be a violation of a right (of the organoid), provided that the organoid is completely passive and has no way of communicating (a bit like an individual with a total locked-in syndrome, whose rights are generally believed to be the same as a healthy individual's). In addition, an entity that has at least a potential for cognitive functions, and thus is in potentia capable of thought, may aspire to be considered a person, since an individual capable of thought will be also capable of rationality-the hallmark of personhood for a number of theories, including the Kantian one. And persons have rights that objects or other forms of life do not have.
Such rights, in particular those to life, respect, and autonomy, are generally considered inalienable. People also have dignity, 'worth beyond value' in Kant's words, although this concept is controversial and its application is not often shared. If a brain organoid were endowed with some features, or properties in a philosophical sense, typical of a person or a person in potentia, this would not mean that it is a person in the usual sense of the term, but surely that it could not be treated as a commodity or a means in the Kantian sense.

It is difficult to imagine what kind of minimal consciousness a cerebral organoid may have, but it is certainly plausible to believe that it can make a difference between its ethical status and that of a mere lump of cerebral tissue. Also from a legal point of view, given that mini-brains are produced starting from donated cells, finding a glimmer of consciousness could open up complex scenarios and make it necessary to use caution in the manipulation and destruction of human organoids.

\section{CONCLUSION}

Human organoids are a major step forward in biomedical research, but they inevitably raise ethical issues about their use. In this sense, it would be helpful to understand the possible implications of the most promising developments of such techniques. The latter have certainly positive purposes and are aimed at the advancement of knowledge and the well-being of people and non-human animals. Nonetheless, the ethical problems associated with the production and use of organoids, especially cerebral organoids, should not be underestimated. It is important to focus on them and exercise an ethical imagination, so to speak, to foresee potential opportunities and risks, without this translating into unnecessary limits for research. The case of organoids is relevant both because biomedical research on the subject, while being still young, is advancing very quickly, and because ethical reflection around it is still yet to be addressed. Indeed, possible future scenarios include the fusion of cerebral organoids to achieve biological structures that are more and more similar to the human brain, endowed with a continuous dorsal-ventral axis and able to model complex interactions between different brain regions. ${ }^{39}$ These are techniques and interventions that could have extraordinary effects on health and care but that also deserve a careful ethical scrutiny ${ }^{\text {ii }}$.

Contributors $\mathrm{AL}$ and $\mathrm{MM}$ contributed equally to planning, conduct and reporting of the work described in the article. They are both responsible for the overall content as guarantors.

Funding This work was supported by European Union's Horizon 2020 Research and Innovation Programme under Grant Agreement 720270 (HBP SGA1), James S. McDonnell Foundation Scholar Award 2013, EU Grant H2020-FETOPEN-2014 -2015-RIA 686764 Luminous and Canadian Institute for Advanced Research, Azrieli Program in Brain, Mind and Consciousness to MM.

Competing interests None declared.

Provenance and peer review Not commissioned; externally peer reviewed.

(c) Article author(s) (or their employer(s) unless otherwise stated in the text of the article) 2018. All rights reserved. No commercial use is permitted unless otherwise expressly granted.

\section{REFERENCES}

1 Putnam H. Brains in a vat. Reason, Truth and History. Cambridge: Cambridge University Press, 1981:1-21.

2 Sato T, Vries RG, Snippert HJ, et al. Single Lgr 5 stem cells build crypt-villus structures in vitro without a mesenchymal niche. Nature 2009;459:262-5.

${ }^{\text {ii }}$ We thank three reviewers for their very helpful comments. 
3 Lancaster MA, Renner M, Martin CA, et al. Cerebral organoids model human brain development and microcephaly. Nature 2013;501:373-9.

4 Cheshire WP. Miniature human brains: an ethical analysis. Ethics Med 2014;30:7-12.

5 Pera MF, de Wert G, Dondorp W, et al. What if stem cells turn into embryos in a dish? Nat Methods 2015;12:917-9.

6 Bredenoord AL, Clevers H, Knoblich JA. Human tissues in a dish: The research and ethical implications of organoid technology. Science 2017:355:eaaf9414.

7 Koch C, Massimini M, Boly M, et al. Neural correlates of consciousness: progress and problems. Nat Rev Neurosci 2016;17:307-21.

8 Tononi G, Sporns 0. Measuring information integration. BMC Neurosci 2003;4:31.

9 Tononi G, Koch C. The neural correlates of consciousness: an update. Ann N Y Acad Sci 2008;1124:239-61.

10 Singer W. Consciousness and the binding problem. Ann N Y Acad Sci 2001;929:123-46.

11 Dehaene S, Changeux JP. Experimental and theoretical approaches to conscious processing. Neuron 2011;70:200-27.

12 Tononi G, Koch C. Consciousness: here, there and everywhere? Philosophical Transactions of the Royal Society B: Biological Sciences 2015;370:20140167.

13 Tononi G, Boly M, Massimini M, et al. Integrated information theory: from consciousness to its physical substrate. Nat Rev Neurosci 2016;17:450-61.

14 Huch M, Koo BK. Modeling mouse and human development using organoid cultures. Development 2015;142:3113-25.

15 Kelava I, Lancaster MA. Stem Cell Models of Human Brain Development. Cell Stem Cell 2016;18:736-48.

16 Huch M, Knoblich JA, Lutolf MP, et al. The hope and the hype of organoid research. Development 2017:144:938-41.

17 Little MH. Organoids: a Special Issue. Development 2017;144:935-7.

18 Qian X, Nguyen HN, Jacob F, et al. Using brain organoids to understand Zika virusinduced microcephaly. Development 2017:144:952-7.

19 Paşca AM, Sloan SA, Clarke LE, et al. Functional cortical neurons and astrocytes from human pluripotent stem cells in 3D culture. Nat Methods 2015;12:671-8.

20 Qian X, Nguyen HN, Song MM, et al. Brain-Region-Specific Organoids Using Minibioreactors for Modeling ZIKV Exposure. Cell 2016;165:1238-54.

21 Lancaster MA, Knoblich JA. Generation of cerebral organoids from human pluripotent stem cells. Nat Protoc 2014;9:2329-40.

22 Birey F, Andersen J, Makinson CD, et al. Assembly of functionally integrated human forebrain spheroids. Nature 2017:545:54-9.
23 Camp JG, Treutlein B. Human development: advances in mini-brain technology. Nature 2017:545:39-40.

24 Li C, Xu D, Ye Q, et al. Zika Virus Disrupts Neural Progenitor Development and Leads to Microcephaly in Mice. Cell Stem Cell 2016;19:120-6.

25 Hub and dutch health insurance. Hub4organoids. 2017 http://hub4organoids.eu/ hub-and-dutch-health-insurance-companies-to-validate-use-of-organoids-forcystic-fybrosis/.

26 Bender E. Science pic: Reprogramming a retina, Novartis. 2015 www.nibr.com/stories/ nerd-blog/science-pic-reprogramming-retina.

27 Ulrich T. Building brain organoids to shed light on disease, Novartis. 2016 www.nibr. com/stories/nerd-blog/building-brain-organoids-shed-light-disease.

28 Giandomenico SL, Lancaster MA. Probing human brain evolution and development in organoids. Curr Opin Cell Biol 2017;44:36-43.

29 Munsie M, Hyun I, Sugarman J. Ethical issues in human organoid and gastruloid research. Development 2017;144:942-5.

30 Quadrato G, Nguyen T, Macosko EZ, et al. Cell diversity and network dynamics in photosensitive human brain organoids. Nature 2017;545:48-53.

31 Massimini M, Boly M, Casali A, et al. A perturbational approach for evaluating the brain's capacity for consciousness. Prog Brain Res 2009:177:201-14.

32 Gosseries $\mathrm{O}, \mathrm{Di} \mathrm{H}$, Laureys $\mathrm{S}$, et al. Measuring consciousness in severely damaged brains. Annu Rev Neurosci 2014:37:457-78.

33 Casali AG, Gosseries O, Rosanova M, et al. A theoretically based index of consciousness independent of sensory processing and behavior. Sci Trans/ Med 2013:5:198ra105.

34 Casarotto $S$, Comanducci A, Rosanova M, et al. Stratification of unresponsive patients by an independently validated index of brain complexity. Ann Neurol 2016;80:718-29.

35 D'Andola M, Rebollo B, Casali AG, et al. Bistability, Causality, and Complexity in Cortical Networks: An In Vitro Perturbational Study. Cereb Cortex 2017:3:1-10.

36 Farah MJ. An ethics toolbox for neurotechnology. Neuron 2015;86:34-7.

37 Godfrey-Smith P. Other Minds: The Octopus, the Sea, and the Deep Origins of Consciousness. New York: Farrar, Straus and Giroux, 2016.

38 Owen A. Into the Gray Zone: A Neuroscientist Explores the Border Between Life and Death. New York: Scribner, 2017.

39 Bagley JA, Reumann D, Bian S, et al. Fusal dorsal-ventral organoids model human cortical interneuron migration. BioRxic 2017. 